Research Article

Qiao Deng, Hui Zhang*, Jun Li, Xuejun Hou, and Hao Wang

\title{
Analysis of impact load on tubing and shock absorption during perforating
}

https://doi.org/10.1515/phys-2019-0022

Received Aug 17, 2018; accepted Mar 18, 2019

\begin{abstract}
During the past few decades, the technologies of the higher-shot densities, larger perforating guns and tubing-conveyed perforation (TCP) combined well testing have been used widely used for well completions. This results in a large increase of impact loads in the tubing during TCP. The safety of the tubing is directly related to the success of perforation combined well test, which is the key link in the oil and gas production. In this study, the influence factors of perforating impact load have firstly been analyzed. Also the dynamic response of tubing during TCP in three dimensions has been studied by numerical simulation. According to the computing results, the vulnerable parts of tubing during TCP have been found, where the axial impact load is the strongest and it is concluded that the axial shock absorber has the optimal installation position to achieve the best shock absorption effect, which is verified by the case. This study proposes a novel method for the safety analysis of the tubing, which has important significance to provide guidance for the design of field perforating operations and to improve security.
\end{abstract}

Keywords: tubing-conveyed perforating, impact load, equivalent stress, shock absorber, numerical simulation

PACS: 02.70.Dh, 07.05.Tp

\footnotetext{
*Corresponding Author: Hui Zhang: China University of Petroleum, Beijing, China; Email: zhanghuicup2018@163.com Qiao Deng: China University of Petroleum, Beijing, China; Email: dengqiao2008@outlook.com

Jun Li: China University of Petroleum, Beijing, China; Email: lijunpaper@hotmail.com

Xuejun Hou: Chongqing University of Science \& Technology, China; Email: xuejun_hou_2013@163.com

Hao Wang: China University of Petroleum, Beijing, China; Email: wh1993china@hotmail.com
} əopen Access. ( 2019 Q. Deng et al., published by De Gruyter. (cc) BY License

\section{Introduction}

Perforating is a critical operation in cased-hole completions where it is necessary to connect the reservoir rock to the wellbore. Technologies of high density perforator, large charge perforating bullet and TCP combined well testing are widely used in field perforation operation. This results in a large increase of the explosion loads on the string. The tool strings and production equipment suffer some damage or destruction, such as bent tubing and upset packers, which seriously affects the normal production of oil and gas and threatens the safety of equipment and personnel [1-4]. Figure 1 shows the tubing buckling caused by perforating impact load during TCP. Therefore, the safety analysis of the string is a critical step in developing perforation designs.

With the worldwide oil and gas exploration and development of deep or ultra-deep well gradually increased, researchers have gradually realized the significance of the study on the impact dynamic effect from explosive loading. Previous studies have found out the factors affecting perforation impact loads. By theoretical analysis and numerical

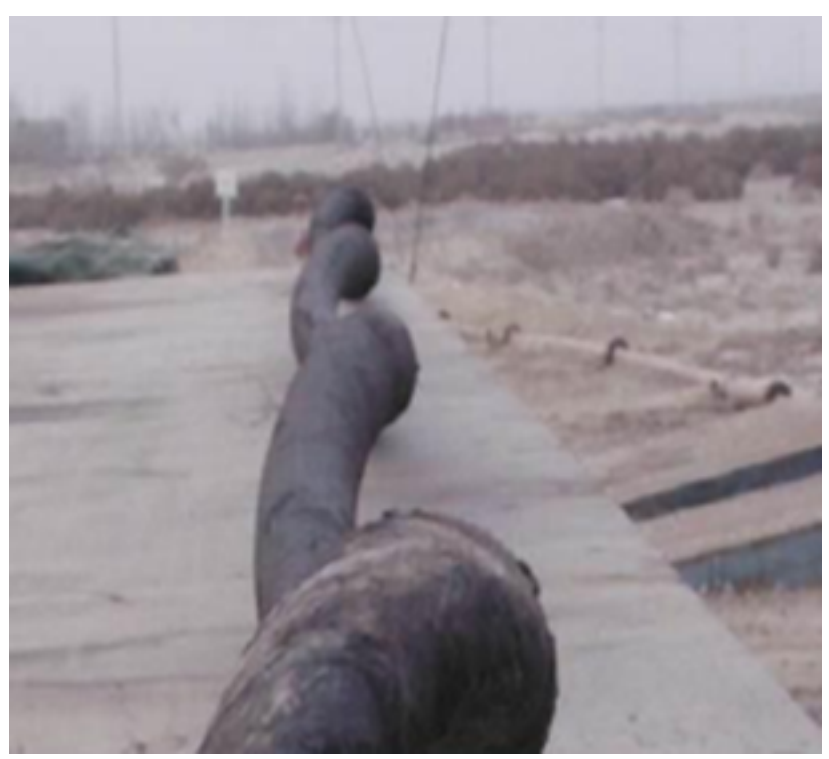

Figure 1: Tubing buckling during TCP 
simulation, the influencing factors of perforation impact load were studied and the methods to effectively reduce the impact load were put forward [5]. It is indicated that the effect of all perforating design elements and dimensions could be analyzed, such as the length of perforated interval and guns loading strategy, types and sizes of guns and shaped charges, cable type and size, wellbore fluid properties and initial pressure, reservoir pressure and properties, position of packers and production tubing size, size and length of conveyance, type and number of shock absorbers and rathole length [6-8]. These studies set a foundation for further research on the shock damage of perforating impact load.

Since 1950s, a lot of experts and scholars have studied the mechanics of tube string and made many achievements. However, the security of the perforated tubing string and its correlative problems gradually turned into the investigative object for researchers in this field in recent years. The static method was used to analyze the perforated pipe string, but only theoretical deduction and qualitative analysis are carried out [9]. The influence factors of perforating shock loads are analyzed by simulation of the finite element software and some methods of alleviating the impact load of perforation was proposed [10]. The perforating community has been developing and exploring the use of numerical models to model dynamic downhole well conditions, all relevant aspects of well perforating were modeled, including gun carrier filling, wellbore pressure waves and associated fluid movement, etc. [11-15]. A model of a multibody system was established, which studied the dynamic response of an oil tube-shock absorber-perforating gun system during perforating. Using the model, the effects of charge quantity of perforating bullet, the number of shock absorbers, and the length of oil tube on the dynamic response of oil tube and packer were investigated [16]. In these studies, the focus was put on the impact load on the string system. However, there is no detailed study on the dynamic response of tubing string and shock absorption, especially for the research on the vulnerable parts.

At present, the methods for perforation research can be classified into three main types: theoretical research, laboratory experiments and numerical simulation. It's hard to fully describe the explosion process through theoretical models for the complicated shock wave loading rule and the dynamic response law of perforated string. In the laboratory research field, it's difficult to stimulate real underground working conditions and it can't really reflect the characteristics of asymmetric dynamic load. Furthermore, the experiment data obtained is limited and the cost is too high. For the numerical simulations, if the model is reasonable, it can fully present the perforating explosion process and simulate various working conditions. The optimization design and proposal can be proposed, which can provide important theoretical basis for field perforating operations.

In this paper, the empirical formula is used to analyze the main influence factors of perforation impact load firstly, and then the dynamic response of the tubing during perforating is simulated, which the vulnerable parts have been found by analyzing the change of the equivalent stress at different positions of tubing and acceleration of $X, Y$ and $Z$ direction at the bottom of the tubing. At last, the corresponding measures are put forward for shock absorption, the field case study show that the best shock absorption effect can be achieved by optimizing the position of the shock absorber. The research has great significance on understanding the influence of impact load on tubing during perforating, providing guidance for the design of perforation, and improving the security of operations.

\section{Influence factors of perforating impact load}

The perforating impact load mainly comes from the energy generated by the high density and certain phase of perforating charge. When the perforating charge explodes in a fluid-filled wellbore, the detonation products rapidly expands in the well in the form of a gas, which will strongly compress the adjacent fluid, causing a sharp increase in pressure and temperature to form an initial pressure wave and to provide the basis condition for dynamic loading in the wellbore, as shown in Figure 2.

Assuming that the initial pressure of the perforation fluid in the wellbore is $P_{0}$ and the pressure increment is $\Delta P$, some energy will be converted into the internal energy of the wellbore fluid after perforation. The pressure of the wellbore fluid after perforation can be expressed as:

$$
P_{+}=P_{0}+\Delta P=P_{0}+\varphi(\delta-1) \frac{n}{V} \frac{m}{M} H
$$

where, $n$ is the number of perforating charge; $V$ is perforated effective volume, $m^{3} ; m$ is single charge, $g$; $M$ is explosive Molar mass, $\mathrm{g} / \mathrm{mol} ; \mathrm{H}$ is explosion heat, $\mathrm{KJ} / \mathrm{mol} ; \delta$ is the hypothetical gas specific heat ratio of the perforating fluid; $\varphi$ is the ratio of the energy of the explosion convert to the internal energy of the perforation fluid.

Without considering formation pressure, it is assumed that the initial pressure of the wellbore fluid is $10 / 50 \mathrm{MPa}$. After perforation, about $30 \%$ of the explosive energy is converted into the fluid internal energy. The number of ammu- 


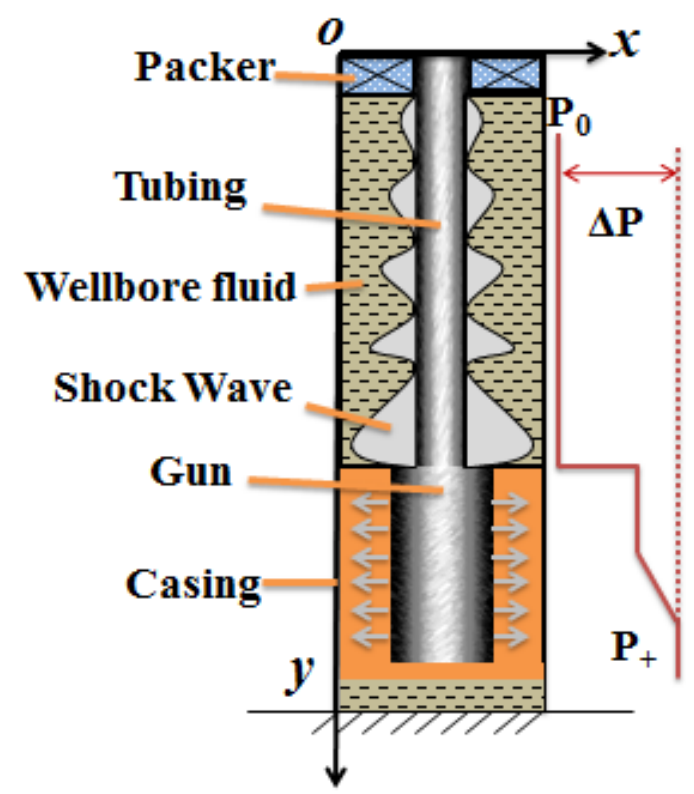

Figure 2: Pressure waves in the wellbore

Table 1: Calculation results

\begin{tabular}{lcccc}
\hline Initial pressure (MPa) & 10 & 10 & 50 & 50 \\
Number of ammunition & 200 & 400 & 200 & 400 \\
\hline Perforating pressure (MPa) & 43 & 76 & 83 & 116 \\
\hline
\end{tabular}

nition is $200 / 400$. Single charge is $40 \mathrm{~g}$, explosive type is $R D X$, casing diameter is $220.5 \mathrm{~mm}$, perforating gun outer diameter is $177.8 \mathrm{~mm}$, length is $9 \mathrm{~m}$. The calculation results are shown in Table 1.

According to the calculation results, it can be known that the number ammunition, the type of perforating charge, the single charge, the structure and the size of the pipe string are the influence factors of the perforating impact load, and the initial wellbore pressure provides a basis pressure for perforation impact load. The number of perforating bullets, the charge and the initial wellbore pressure have the greatest influence on the impact load of perforation.

The dynamic response of a perforated string under the explosive impact load is a cross-scale and strongly nonlinear dynamic problem. It responds ranging from material elasticity to plasticity and also includes complex yield conditions, strain hardening and strain rate effects. In addition, the large deformation is often involved in the problem. In the finite element theory [17], the basic equation of dynamics is as follows:

$$
[M]\left\{x^{\prime \prime}\right\}+[C]\left\{x^{\prime}\right\}+[K]\{x\}=\{F(t)\}
$$

where, $[M]$ is the mass matrix; $[C]$ is the damping matrix; $[K]$ is the stiffness matrix; $\{F(t)\}$ is the external load varying with time; $\left\{x^{\prime \prime}\right\},\left\{x^{\prime}\right\}$, and $\{x\}$ are the acceleration, velocity and displacement vectors of the node, respectively.

Compared to statics, external loads over time and the inertia and damping of the media can make the problem more complicated. A large number of dynamic experiments show that solid materials usually have the following dynamic characteristics and the yield strength has improved significantly. The instantaneous stress increases with the increasing strain rate and decreases with the increase of temperature. Among them, the phenomenon of the yield strength and instantaneous stress increase with the increasing strain rate is known as the strain rate effect, which is the main aspect of the dynamic properties of solid materials.

The output law of perforation explosion load and the dynamic response of the string are extremely complex, thus, it is hard to fully describe them with the theoretical models. The whole process involves the detonation of explosives, the formation of jet and the coupling of fluid and solid and many other phenomena. The impact load acting on the string is asymmetric and uneven. The detonation flow field has extremely complicated coupling and superposition effects, which persist in a certain time range and increase the complexity of the impact load on the string, resulting in the complicated stress and strain state of the pipe string under the extremely complex and strong impact load. Therefore, the finite element software of ANSYS/LS-DYNA can be used to study the whole dynamic process accurately.

\section{Numerical simulation}

\subsection{Modeling and meshing}

In the actual perforation operation, different lengths of column will be used under different well conditions. The column has different components, so a certain simplification of the column system is to be done. The simplified model of TCP is shown in Figure 3. The model mainly consists of perforating gun, tubing and casing, with a certain phase and number of perforating bullets distributed in the perforating gun. The remaining space inside the gun is filled with air, the tubing and annulus are filled with well fluid, the upper end of the tubing is radially restrained by the packer, the lower portion is restricted by the well bottom, and the surrounding is confined by the casing. 


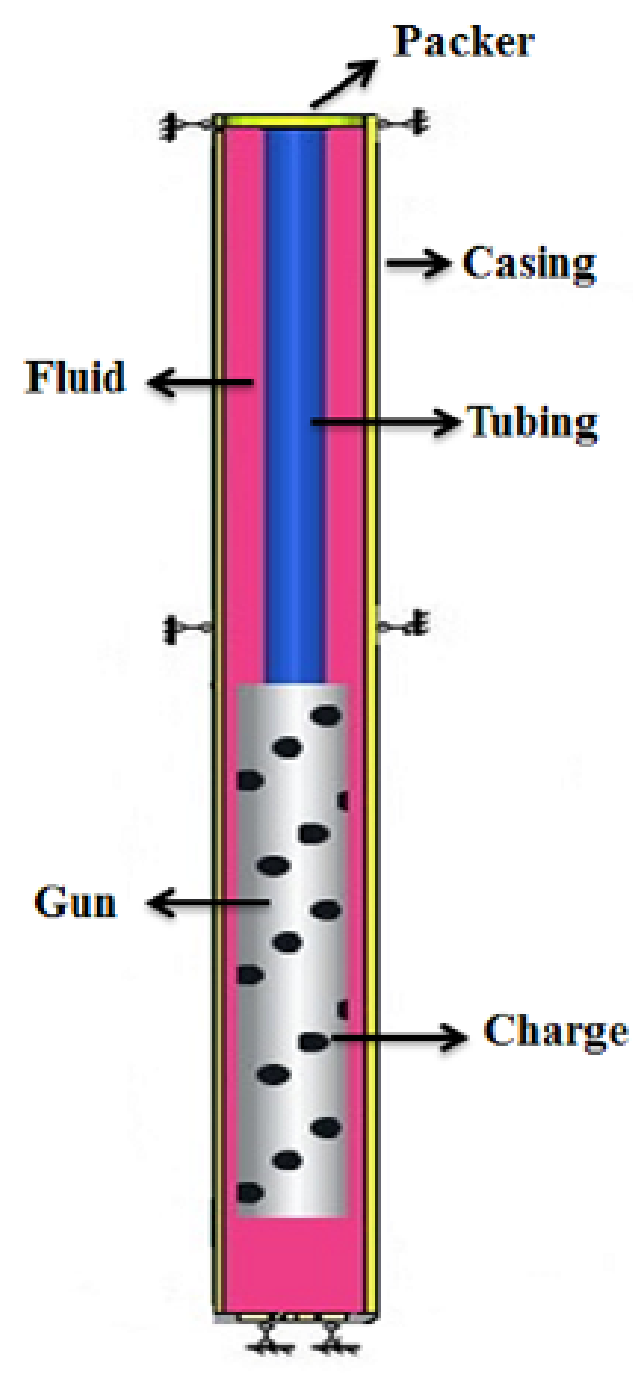

Figure 3: Simplification of the string system

According to the simplified perforation string system, the 3D physical model can be established: the perforating gun is $6 \mathrm{~m}$, the tubing is $7 \mathrm{~m}$, the down-hole pocket is $6 \mathrm{~m}$; the outer diameter of the casing is $177.8 \mathrm{~mm}$ and the wall thickness is $10.4 \mathrm{~mm}$; the outer diameter of the perforating gun is $127 \mathrm{~mm}$ and the wall thickness is $11 \mathrm{~mm}$; the outer diameter of the tubing is $73.02 \mathrm{~mm}$ and the wall thickness is $7.01 \mathrm{~mm}$; the steel grade of casing and tubing are N80, its yield limit is $460 \mathrm{MPa}$ and $536 \mathrm{MPa}$, respectively. The perforating parameters are as follows: the hole density is 30 holes per meter, the single charge is $37 \mathrm{~g}$, the phase angle is $90^{\circ}$, the perforating bullet is detonated in time series, with the time range of $O$ to $640 \mu \mathrm{s}$.

Lagrange algorithm is used in perforating gun, tubing and casing. The arbitrary Lagrangian-Eulerian (ALE) is used in explosives, air and liquid, which can alleviate many of the drawbacks that the traditional Lagrangian- based and Eulerian-based finite element simulations have. When using the ALE technique in engineering simulations, the computational mesh inside the domains can move arbitrarily to optimize the shapes of elements, while the mesh on the boundaries and interfaces of the domains can move along with materials to precisely track the boundaries and interfaces of a multi-material system. ALE-based finite element formulations can reduce to either Lagrangian-based finite element formulations by equating mesh motion to material motion or Eulerian-based finite element formulations by fixing mesh in space [18]. The calculation model is divided by hexahedron grid. In order to capture the movement and deformation of the construction of the material effectively and ensure energy between the partial grid effective transmit, each part of the material on the joined interface must have the common mesh node, as shown in Figure 4. The mean grid spacing is $4-5 \mathrm{~mm}$ and the total number of grids is about 1 million.

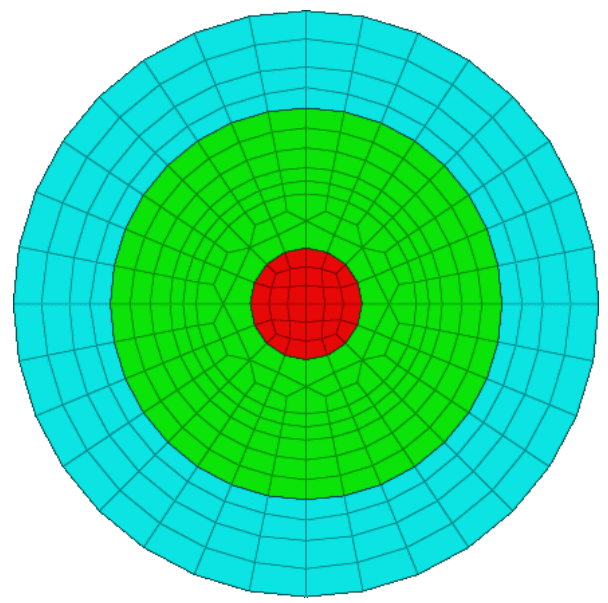

Figure 4: Grid partition

The $R D X$ is used for perforating charge, and the non-linear plastic kinematic hardening model PLASTIC_KENEMATIC is adopted for the column material. Assuming that the fluid zones are connected to the same node, the fluid is coupled to the solid interface and the unit algorithm adopts the constant stress unit algorithm of SOLID164 unit. The material model of the charge is highenergy dynamite HIGH_EXPLOSIVE_BURN, the state equation is EOS_JWL [19], which can accurately describe the pressure, volume and energy characteristics of detonation products in the process of explosion driving. It can be expressed as:

$$
p=A\left(1-\frac{\omega}{R 1 V}\right) e^{-R 1 V}+B\left(1-\frac{\omega}{R 2 V}\right) e^{-R 2 V}+\frac{\omega E}{V}
$$


where, $V$ is the relative volume; $E$ is the initial internal energy per unit volume of charge, $J ; A, B, w, R_{1}, R_{2}$ are the physical parameters of charge.

When the perforating charge is detonated, the high pressure detonation gas is generated and rapidly diffuses in the perforation gun. On the one hand, the shock wave will directly affect the barrel and pass the load through the barrel to the shock absorbers, tubing, screen, packer and other string structures, causing a strong impact vibration of the tubing string system. On the other hand, the impact load will cause the liquid pressure outside the column to change drastically in a short time, and then spread as shock wave in the perforating fluid. This will instantly cause large deformation of the liquid and move rapidly and violently in the well, affecting the structural stability of the column system and local structural strength [20-22].

\subsection{Computing results}

Under the strong impact load and fluid-structure interaction, perforated pipe string will be in a very complex state of stress and strain. Experimental testing or perforation software usually cannot visually display how the stress or strain changes and where is the vulnerable parts of the tubing. From the nephogram calculated by numerical simulation, it is visualized to see how the equivalent stress of the tubing changes during the perforation process, as shown in Figure 5.

It can be seen the equivalent stress of the tubing presents a periodic change. After the detonation of the perforating shaped charge, the bottom of the tubing responds firstly and the equivalent stress rises rapidly. The stress wave is transmitted upward the tubing and the energy is gradually absorbed by the tubing, which finally be-

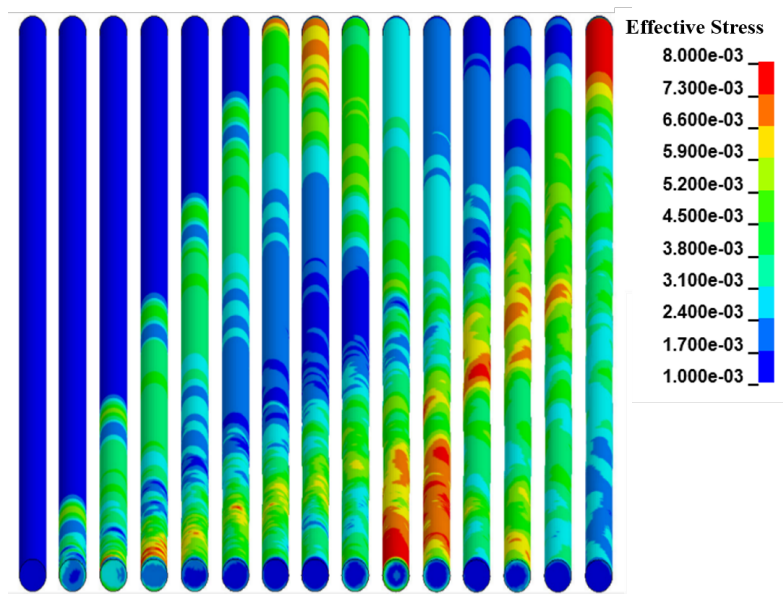

Figure 5: Equivalent stress of tubing during perforating come the strain energy of the tubing. The equivalent stress reaches the extreme value at the top of the tubing where is the fixed end. Subsequently, the stress wave reflects downwards and reaches the maximum at the bottom, which is one cycle. Stress wave reflects at the bottom of the tubing and it will enter the next cycle. Stress wave propagation in the pipe back and forth, making the maximum equivalent stress alternating in all parts of the tubing. At about 1500 $\mu \mathrm{s}$, the equivalent stress at the top of the tubing reaches the maximum for the first time; at about $3500 \mu \mathrm{s}$, the equivalent stress at the top of the tubing reaches the maximum for the second time.

Figure 6 shows the trend of the maximum equivalent stress on the tubing changing over time. It can be concluded that the maximum equivalent stress appears at the top and bottom of the tubing, the pressure wave cannot propagate further as the top and bottom is restricted. The pressure wave is reflected and enhanced at these two positions. From the aspect of the change trend of equivalent stress, it can be known that the maximum equivalent stress still shows periodic variation, which fluctuates back and forth between the bottom and the top of the tubing. According to the numerical value of the equivalent stress, the fluctuation amplitude of the equivalent stress is roughly the same in the first two cycles. In the third cycle, the maximum equivalent stress rapidly decreases after a larger extreme occurring at the top of the tubing. Then the maximum equivalent stress does not rise anymore. Through the analysis above, the maximum equivalent stress repeatedly appears at the bottom and top of the tubing, which can be assessed as vulnerable parts. Therefore, the safety design for these two positions should be deliberately considered for a perforation operation.

The impact overload caused by the perforating shaped charge explosion is transmitted to the perforated pipe

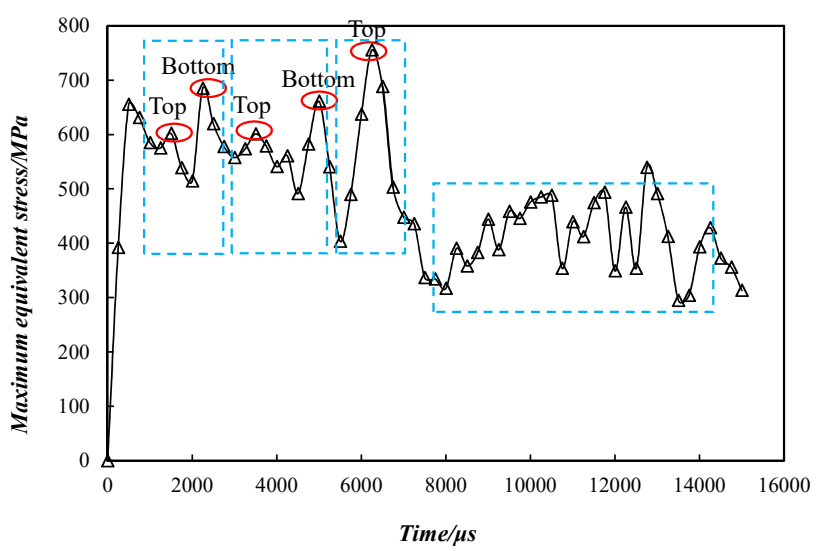

Figure 6: Maximum equivalent stress time-varying curve 
string through the perforating gun. The tubing is subjected to tensile or compressive loads in the axial direction, shear loads in the radial direction and torsional loads in the circumferential direction. In the process of field perforation, those three types of impact loads may alternately and repeatedly act on the pipe string. As the multiple lamination caused by the pressure difference between the inner and the outer wall of the pipe string, a complex kinetic response may occur to the perforated string. The bottom of the tubing is screwed with the perforating gun, which can be regarded as the initial output unit of the explosive load. Dynamic analysis of the bottom of the tubing and the perforating gun is the basis for further analysis of the dynamic damage of the perforated pipe string. Therefore, the response rules can be obtained by analyzing the kinetic data at the bottom of the tubing. However, dynamic calculation is accompanied by stress wave refraction and reflection, which leads to the dynamic data of different nodes varying greatly. In order to accurately analyze the output form and law of the explosive load, a structural block (part) at the bottom of the tubing is built to analyze its dynamic response law during the explosion of the perforating shaped charge.

Figure 7 illustrates the axial acceleration time curve at the bottom of the tubing. The small figure is a scaled view in the range of 0 to $700 \mu \mathrm{s}$. In the first $700 \mu \mathrm{s}$ (the process of an explosion), the response of acceleration is the most obvious and shows a trend of shock decay. Under the impact of explosion load, the positive and the negative peaks of the acceleration at the bottom of the tubing are $2.21 \times 104 \mathrm{~g}$ (gravity acceleration) and $-1.69 \times 104 \mathrm{~g}$, respectively. Under such a strong cyclical axial impact, the tubing will show buckling instability or even fracture in the macroscopic scale.

Figures 8 and 9 are the acceleration time curves of radial $X$ and radial $Y$ at the bottom of the tubing, respectively.

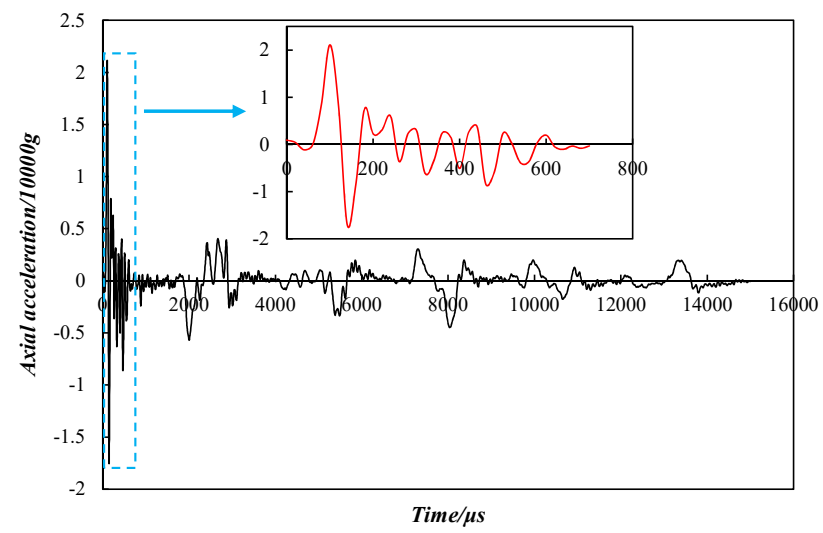

The small figure is a scaled view in the range of 0 to 700 $\mu s$ (the detonation process of perforating shaped charge). The radial acceleration of $X$ and $Y$ has several peaks during the detonation process of perforating shaped charge. It is mainly because the shock wave of the explosion oscillates back and forth in a narrow space downhole. Continuous impact is applied on the perforating gun in the radial direction. Different from the case of radial acceleration, there is more space for detonation gas expansion in the axial direction, which mitigates the continuous impact applied on the perforating gun. Under the impact of explosion load, the positive and the negative peak values of $X$ radial acceleration at the bottom of the tubing is $1.53 \times 104 \mathrm{~g}$ and $-1.01 \times 104 \mathrm{~g}$, respectively. The tubing usually oscillates due to the changing radial load, resulting in a significant shear load on the tubing.

Table 2 shows the acceleration peaks of the tubing in $X, Y$ and $Z$ directions. Analyzed together with the acceleration curve, the greatest dynamic response appears in the axial direction, followed by the $X$ direction, and the $Y$ direction at last, while there is little difference between $X$ and $Y$ directions.

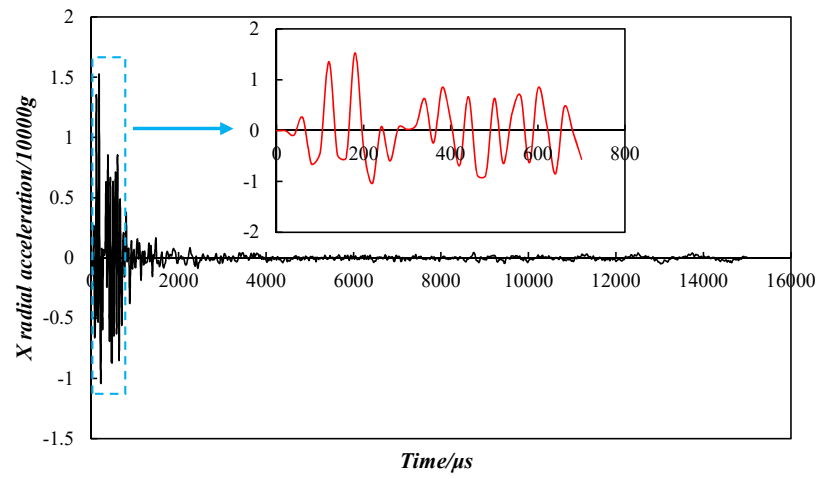

Figure 8: $X$ radical acceleration time-varying curve

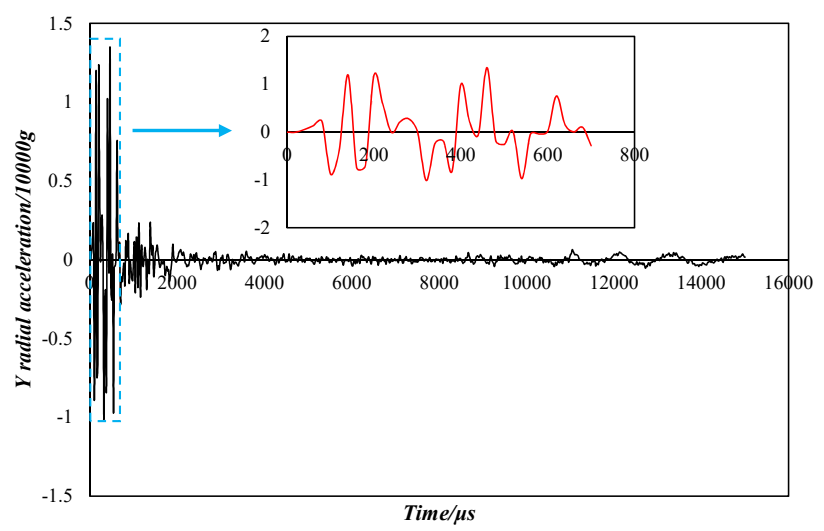

Figure 9: $Y$ radical acceleration time-varying curve

Figure 7: Axial acceleration time-varying curve 
Table 2: Calculation results

\begin{tabular}{ccc}
\hline Direction & Peak $(+) / 10000 \mathrm{~g}$ & Peak $(-) / 10000 \mathrm{~g}$ \\
\hline Axial & 2.21 & -1.69 \\
$\mathrm{X}$ radical & 1.53 & -1.04 \\
$\mathrm{Y}$ radical & 1.35 & -1.01 \\
\hline
\end{tabular}

Therefore, it is necessary to install a longitudinal shock absorber below the packer in a well to reduce the impact vibration on it and the upper instruments.

\section{Shock absorption}

The axial shock absorber with spring element is used to achieve the best damping effect. In order to study the installation position, it is assumed that the distance between the shock absorber and the perforating gun is $R_{0}$ and the ratio of the distance between the shock absorber and the perforating gun to the distance of the packer is $R_{0} / R$, as shown in Figure 10.

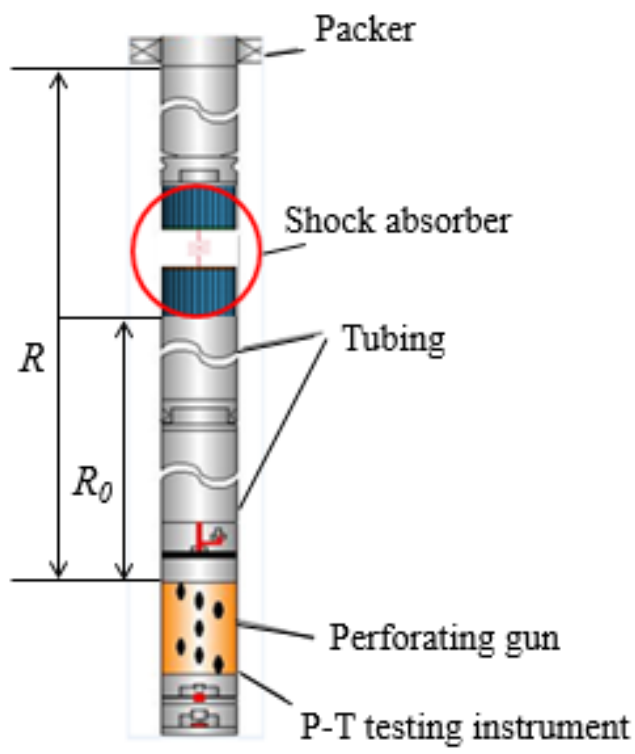

Figure 10: Installation position of the shock absorber

The numerical simulation method is similar to Section 3.1. The model needs to be simplified, the shock absorber is simplified as mechanical spring. The calculation model is divided by hexahedron grid. Lagrange algorithm is used in the perforated string, ALE algorithm is used in explosives, air and liquid. The non-linear plastic kinematic hardening model (PLASTIC_KENEMATIC) is adopted for the column material. The material model of the charge is HIGH_EXPLOSIVE_BURN, the state equation is JWL, the unit algorithm adopts the constant stress unit algorithm of SOLID164 unit.

Based on the actual parameters of the field example in South China Sea [23], the number of perforating bullets is 336 , single charge is $40 \mathrm{~g}$, the distance from the packer to the top of the perforator gun length is $19.14 \mathrm{~m}$, the gun length is $6 \mathrm{~m}$, the initial wellbore pressure is $10 \mathrm{MPa}$ and the formation pressure is $12 \mathrm{MPa}$. Through numerical simulation, the curve of the axial force reduction of the string can be obtained for different numbers of shock absorbers. The results are shown in Figure 11.

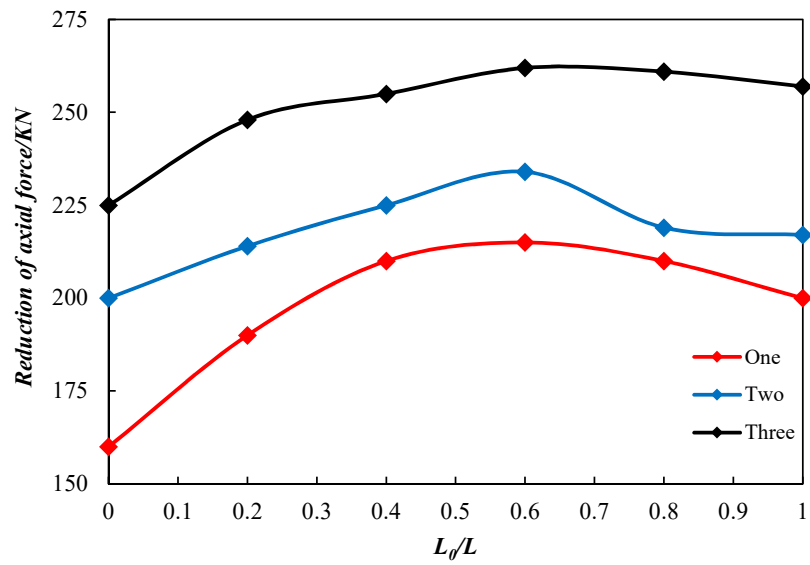

Figure 11: Axial force reduction of the string under different installation position

The figures reveal that the shock absorbers have the optimal installation position. When the ratio $R_{0} / R=0.6$, all of the three cases for different number of the shock absorbers have the maximal axial force reduction of 215, 234, $262 \mathrm{KN}$, respectively. The field application shows that it can achieve the best damping effect by optimizing the setting position of the shock absorbers.

\section{Conclusion}

The theory analysis shows that the number of bullets, the charge, the size of the pipe string, the initial wellbore pressure are the factors affecting perforating impact load. The basic equation of the dynamic response of the perforated string have been proposed. The numerical simulation analysis presents a new method of modeling and meshing, which has solved the complexity of the whole dynamic 
process during TCP and ensured the accuracy and reasonableness of the simulation results. The dynamic threedimensional response process of perforated tubing is presented by numerical simulation.

The results show that the extreme values of maximum equivalent stress appear at the top and bottom of the tubing, which can be regarded as the vulnerable parts, avoiding damage by increasing the steel grade or wall thickness of the tools installed on it. The tubing has the greatest dynamic response in the axial direction, followed by the $X$ radial and the $Y$ radial. It is recommended to use axial shock absorbers to reduce the impact on the string system. According to the numerical simulation results of the field example in South China Sea, the shock absorbers have the optimal installation position, when the ratio $R_{0} / R$ is 0.6 , so that the best shock absorption effect can be achieved.

This paper proposes a novel method for studying the impact load on the string and shock absorption during TCP, which has important significance to provide guidance for the design of TCP operations and to improve security. However, more different perforation conditions need to be simulated and the results need to be verified by more field examples.

Acknowledgement: The authors gratefully acknowledge the Natural Science Foundation of China (Grant No. U1762211, Grant No. 51734010, Grant No. 51574262, Grant No.51774304, Grant No. 51774063), National Oil and Gas Major Project (Grant No. 2017ZX05009), the Foundation for Innovative Research Groups of the National Natural Science Foundation of China (Grant No. 51521063), the State Key Laboratory of Petroleum Resources and Engineering.

\section{References}

[1] Eichelberger R.J., Experimental test of the theory of penetration by metallic jets, J. Appl. Phys., 1956, 27, 1, 63-68.

[2] Lee W.H., Oil well perforator design using 2D Eulerian code, Int. J. Impact. Eng., 2002, 27, 5, 535-559.

[3] Grove B., Werner A., Han C., Explosion-induced damage to oilwell perforating gun carriers, In: Structures Under Shock and Impact IX Conference, 2006, 165-176.

[4] Elshenawy T., Li Q.M., Influences of target strength and confinement on the penetration depth of an oil well perforator, Int. J. Impact. Eng., 2013, 54, 130-137.

[5] Chen H., Tang K., Ren G., String dynamic mechanics analysis on ultra-deep perforation, Well. Logging. Techno., 2010, 34, 5, 487-491.

[6] Zhang W., Xie S., Lu Q., Influencing analysis of perforation impact load on combined string of perforation-acidification-test, Well. Testing., 2016, 25, 6, 8-71.
[7] Baumann C.E., Bustillos E.P., William A., Williams H.A.R., Reduction of perforating gunshock loads. In: Brasil Offshore Conference and Exhibition (14-17 June 2011, Macaé, Brazil), Brazil, 2011, 1-10.

[8] Bale D.S., Satti R.P., Ji M., Howard, J.J., A Next-Generation ShockCapturing, Multi-Phase Flow Simulator for Perforating Applications in HPHT Environment. In: SPE Deepwater Drilling \& Completions Conference (14-15 September 2016, Galveston, Texas), Texas, USA, 2016, 10-18.

[9] Zhang J., Li S., Mechanics analysis of perforating combined well testing string and protection technology of downhole instrument, Oil. Drilling. Prod. Techno., 2003, 25, 3, 61-63.

[10] Chen F., Chen H., Tang K., Influence of perforating impact load on the operating string and the countermeasures, Nature. Gas. Ind., 2010, 30, 5, 61-65.

[11] Canal A. C., Miletto P., Schoener-Scott M. F., Medeiros J., Barlow D., Predicting pressure behavior and dynamic shock loads on completion hardware during perforating. In: Offshore Technology Conference, January, 2010, 125-138.

[12] Sanders W., Baumann C.E., Williams H.A.R., Moraes F.D., Shipley J., Bethke M.E., Efficient Perforation of High-Pressure Deepwater Wells. In: Offshore Technology Conference, Houston, Texas, USA, 2-5 May, 2011, 22-36.

[13] Baumann C., Dutertre A., Martin A., Williams H., Risk Evaluation Technique for Tubing-Conveyed Perforating. In: EAGE Annual Conference \& Exhibition incorporating SPE Europec, Copenhagen, Denmark, 4-7 June, 2012, 33-45.

[14] Baumann C.E., Lazaro A., Valdivia P., Williams H., Stecchini P., Perforating Gunshock Loads - Prediction and Mitigation. In: SPE/IADC Drilling Conference and Exhibition, Amsterdam, The Netherlands, 5-7 March, 2013, 56-68.

[15] Baumann C.E., Brinsden M.S., Perforating Gunshock Loads: Simulation and Optimization in 2014. In: IADC/SPE Asia Pacific Drilling Technology Conference, Bangkok, Thailand, 25-27 August, 2014, 199-212.

[16] Liu J., Li S., Liu Q.Y., Guo X.Q., He Y.F., Study on dynamic response of downhole tools under perforation impact load, Shock. Vib., 2017, 1-11.

[17] Ning J.G., Wang C., Ma T.B., Explosion and Shock Dynamics. National Defense Industry Press, Beijing, 2010.

[18] Teng Y.S., Dynamic Simulation for Perforating and String Safety Evaluation, Master thesis, China University of Petroleum (East China), Qingdao, 2014.

[19] Deng Q., Zhang H., Li J., Wang H., Cai Z.X., Tan T.Y., et al., A Model for Estimating Penetration Length Under Different Conditions. In: 52nd U.S. Rock Mechanics/Geomechanics Symposium, 17-20 June, Seattle, Washington USA, 2018, 10-28.

[20] Cai L.Z., Zhao X., Xue S.F., Yang Z.Z., Dynamic Response Analysis of String Structure during Perforating Process. Oil. Field. Equip., 2015, 44, 5, 26-30.

[21] Zhou H.F., Research on the Dynamic Response of the Oil Well Perforating String to Explosive Impact Load, Master thesis, Beijing Institute of Technology, Beijing, 2014.

[22] Wang R., Gao X., Song H., Shang X., Analytical modeling of coupled flow and geomechanics for vertical fractured well in tight gas reservoirs, Open. Phys., 2017, 15, 1, 797-802.

[23] Deng Q., Zhang H., Li J., Dong J., Wang H. Hou X.J., Safety Distances of Packers for Deep-Water Tubing-Conveyed Perforating. In: Offshore Technology Conference (30 April-3 May 2018, Houston, Texas), Texas, USA, 2018, 1-10. 